\title{
Why tensors should be taught at undergraduate levels
}

\author{
Andrey Beyle, Bernard Maxum
}

Andrey Beyle, Bernard Maxum, "Why tensors should be taught at undergraduate levels," Proc. SPIE 9793, Education and Training in Optics and Photonics: ETOP 2015, 979319 (15 December 2015); doi:

$10.1117 / 12.2223104$

SPIE Event: Education and Training in Optics and Photonics: ETOP 2015, 2015, Bordeaux, France 


\title{
Why tensors should be taught at undergraduate levels \\ by Andrey Beyle* and Bernard Maxum** \\ Lamar University
}

\begin{abstract}
Many academic subjects that were taught previously in the framework of theoretical physics moved to engineering. These include courses in electromagnetics, statics and dynamics, heat and mass transfer, mechanics of solids, nuclear power, and courses that branch from these, like fiber optic communications thermodynamics. However, the mathematical foundation in engineering education has remained substantially unchanged during this transition period, typically peaking at the level of linear algebra, vector calculus and integral transforms. As a result many undergraduate engineering courses are built in such a way as to avoid tensor analysis and tensor calculus, as if such mathematical constructs are beyond the capacity of the undergraduate student to understand. We show that this not the case.
\end{abstract}

This paper is devoted to demonstrating examples of phenomena studied in engineering courses that are actually more easily explained based on tensor nature of participating factors. These examples are taken from optics/ photonics applications by formulating their respective constitutive relationships with tensorial field quantities as a natural extension to what they already know in vector analysis. We show the tensorial relationships become quite clear and workable for the undergraduate student. Examples include birefringence, photoelasticity, nonlinear optics, physics and mechanics of composites and metamaterials.

With these examples we show why tensor algebra and tensor calculus should be taught as a part of vector algebra and vector calculus, thus obviating the necessity for students to have to un-learn material before the being ready to begin working in optics, photonics, photoelasticity mechanics of composites and metamaterials. This approach uses students' time more efficiently.

To test these suppositions we used tensor concepts in teaching the following subjects:

- Statics and Dynamics

- Mechanics of Solids

- Mechanics of Composite Materials and Structures

- Engineering Electromagnetics,

- Advanced Electromagnetics

- Optical Communications

- Fiber Optic Systems

- Nonlinear Fiber Optics

- Advanced Engineering Mathematics

Tensors were introduced under three points of view:

- as guaranteed invariant objects in rotation of the system of coordinates having law of components transform in the form of a generalization of the corresponding law for vectors

- $\quad$ as objects with rank $r$ linearly connecting two objects with ranks $p$ and $q$, where $r=p+q$ and with initial ranks $r=0$ for scalar, $r=1$ for vector objects, and $r=2$ for dyadics, etc;

- $\quad$ as a results of different multiplications of vectors (forming tensors of varying rank).

Let's consider some examples of the modern analytical methods as a first exposure to tensors for undergraduate students.

Education and Training in Optics and Photonics: ETOP 2015, edited by Eric Cormier, Laurent Sarger Proc. of SPIE Vol. 9793, 979319 - (C) 2015 SPIE, IEEE, OSA, ICO · doi: 10.1117/12.2223104 


\section{Example 1. Constitutive Relationships between Electric and Magnetic Fields}

Introductory courses dealing with electric and magnetic fields typically begin with homogeneous isotropic material media when the field intensities are at a sufficiently low level such that the medium remains linear with incremental increments in field strength. In these simplistic cases the flux density field vectors $\vec{D}$ and $\vec{B}$ are linearly related to their respective vector field intensities $\vec{E}$ and $\vec{H}$ by scalar permittivity and permeability constants $\varepsilon_{o}$ and $\mu_{0}$, respectively. These are traditionally expressed in introductory EM courses by the constitutive expressions $\vec{D}=\varepsilon_{0} \vec{E}$ and $\vec{B}=\mu_{0} \vec{H}$, where we show vector fields with the single-headed arrows " $\rightarrow$ " over bars. However, for anisotropic media the constitutive relations become

$$
\begin{aligned}
\vec{D} & =\overleftrightarrow{\varepsilon} \cdot \vec{E} \text { and } \\
\vec{B} & =\overleftrightarrow{\mu} \cdot \vec{H}
\end{aligned}
$$

And combining these as

$$
\left(\begin{array}{l}
\vec{D} \\
\vec{B}
\end{array}\right)=\left(\begin{array}{ll}
\overleftrightarrow{\varepsilon} & 0 \\
0 & \overleftrightarrow{\mu}
\end{array}\right) \cdot\left(\begin{array}{l}
\vec{E} \\
\vec{H}
\end{array}\right)
$$

where we show the permittivity and permeability as nine-component dyadics $\overleftrightarrow{\varepsilon}$ and $\overleftrightarrow{\mu}$ with the double-headed arrow over bars to denote the "dual directional compoundedness" as described in Field Mathematics ${ }^{1}$, Section 3.1. This is often the first exposure for students that there are quantities that require "rank" values greater than one.

When vectors were first mentioned at the freshman-sophomore level, instead of defining vectors as quantities that have direction and magnitude in order to distinguish them from scalars that have magnitude only, the word "single" should be added ${ }^{2}$, namely "vectors are quantities that have magnitude and a single direction." With emphasis on the word single, this implants the image on the student at an early stage that there are quantities that have multiple directionality and that such quantities are not three-component vectors. Thus $\overleftrightarrow{\varepsilon}$ and $\overleftrightarrow{\mu}$ are ninecomponent dyadics described by

$$
\begin{aligned}
\left(\begin{array}{c}
\vec{\varepsilon} \\
\vec{\mu}
\end{array}\right) & =\hat{u}_{1} \hat{u}_{1}\left(\begin{array}{l}
\varepsilon_{11} \\
\mu_{11}
\end{array}\right)+\hat{u}_{1} \hat{u}_{2}\left(\begin{array}{c}
\varepsilon_{12} \\
\mu_{12}
\end{array}\right)+\hat{u}_{1} \hat{u}_{3}\left(\begin{array}{c}
\varepsilon_{13} \\
\mu_{13}
\end{array}\right) \\
& +\hat{u}_{2} \hat{u}_{1}\left(\begin{array}{l}
\varepsilon_{21} \\
\mu_{21}
\end{array}\right)+\hat{u}_{2} \hat{u}_{2}\left(\begin{array}{c}
\varepsilon_{22} \\
\mu_{22}
\end{array}\right)+\hat{u}_{2} \hat{u}_{3}\left(\begin{array}{c}
\varepsilon_{23} \\
\mu_{23}
\end{array}\right) \\
& +\hat{u}_{3} \hat{u}_{1}\left(\begin{array}{l}
\varepsilon_{31} \\
\mu_{31}
\end{array}\right)+\hat{u}_{3} \hat{u}_{2}\left(\begin{array}{c}
\varepsilon_{32} \\
\mu_{32}
\end{array}\right)+\hat{u}_{3} \hat{u}_{3}\left(\begin{array}{c}
\varepsilon_{33} \\
\mu_{33}
\end{array}\right) \\
& =\sum_{i=1}^{3} \sum_{j=1}^{3} \hat{u}_{i} \hat{u}_{j}\left(\begin{array}{c}
\varepsilon_{i j} \\
\mu_{i j}
\end{array}\right)=\sum_{i=1}^{3} \sum_{j=1}^{3} \widehat{u_{i j}}\left(\begin{array}{c}
\varepsilon_{i j} \\
\mu_{i j}
\end{array}\right)=\left(\begin{array}{c}
\leftarrow 2 \rightarrow \\
\varepsilon \\
\leftarrow 2 \rightarrow \\
\mu
\end{array}\right)
\end{aligned}
$$


Here we point out to the undergraduate that the nine unit vector combinations $\hat{u}_{i} \hat{u}_{j}$ are bidirectional unitary dyadics and that without a dot or cross product operation between them one should think of them as a single entity which we refer to as a unitary dyadics. We explain in Reference 1, Section 3.1 that dual directional compoundedness in these cases refers to the direction of the applied field $\vec{E}$ or $\vec{H}$ and the resultant flux density fields $\vec{D}$ or $\vec{B}$ directions that in general differ when the medium is anisotropic.

In writing the bidirectional unitary dyadic $\hat{u}_{i} \hat{u}_{j}$, with neither dot or cross product operations implied between the unit vectors $\hat{u}_{i}$ and $\hat{u}_{j}$, the unitary dyadic is a single entity and thus we might prefer to write it as $\hat{\hat{u}}_{i j}$ to emphasize that situation. Thus,

$$
\hat{u}_{i} \hat{u}_{j} \equiv \hat{\hat{u}}_{i j}
$$

In the second to last equality of (1-4) the explicit representation $\hat{\hat{u}}_{i j}\left(\begin{array}{c}\varepsilon_{i j} \\ \mu_{i j}\end{array}\right)$, where the nine components of the dyadics $\left(\begin{array}{c}\overleftrightarrow{\varepsilon} \\ \overleftrightarrow{\mu}\end{array}\right)$, is represented by the scalar magnitude coefficients $\left(\begin{array}{c}\varepsilon_{i j} \\ \mu_{i j}\end{array}\right)$ in the bidirection $\hat{\hat{u}}_{i j}$. In Field Mathematics ${ }^{3}$ the term "dyad" is coined to describe this unitary dyadic.

Notice that the last equality of (1-4) has a "2" inserted in the double-headed arrow over bar in order to emphasize another more explicit depiction of the dual directionality of the two-rank tensors (dyadics) $\stackrel{\leftarrow 2 \rightarrow}{\varepsilon}$ and $\stackrel{\leftarrow 2 \rightarrow}{\mu}$. This allows for later depicting higher-rank tensors beyond the dyadic with equal simplicity of notation, which we have found is readily understood by the undergraduate student.

\section{Example 1a. Generalization to metamaterials}

In this next example we generalize the prior example to include metamaterials where the constitutive expressions take the form $\vec{D}=\vec{\varepsilon} \cdot \vec{E}+\vec{\varsigma} \cdot \vec{H}$ and $\vec{B}=\vec{\mu} \cdot \vec{H}+\vec{\xi} \cdot \vec{E}$ and where $\vec{\varsigma}$ and $\vec{\xi}$ are the cross field dyadics. As before we combine these expressions

$$
\left(\begin{array}{l}
\vec{D} \\
\vec{B}
\end{array}\right)=\left(\begin{array}{ll}
\overleftrightarrow{\varepsilon} & \vec{\zeta} \\
\vec{\xi} & \overleftrightarrow{\mu}
\end{array}\right) \cdot\left(\begin{array}{l}
\vec{E} \\
\vec{H}
\end{array}\right)
$$

which is a generalization of (1-3).

With these examples of dyadics-rank two tensors-provided to our undergraduate students, we next introduce third-rank tensors. 


\section{Example 2. Piezoelectric and magnetoelasticity effects}

The piezoelectric effect ${ }^{4}$ is understood as the linear electromechanical interaction between the mechanical stress and the applied electric field intensity in crystalline materials with no inversion symmetry ${ }^{5}$. Whenever such a material is immersed in an electric field $\vec{E}$, the material encounters an internal stress described by the dyadic $\vec{\sigma}$ as

$$
\vec{E}=\stackrel{\leftrightarrow 3}{\Psi}: \overleftrightarrow{\sigma}
$$

where $\stackrel{\leftrightarrow 3}{\Psi}$ is the third rank piezoelectric stress triadic, which is a 27 component rank three tensor ${ }^{6}$. Its 27 components contain only 18 independent values for general anisotropic crystals. The piezoelectric effect is a reversible process in that there is an internal generation of an electric field when a mechanical strain is applied from an external force.

Magnetostriction $^{7}$ is a property of ferromagnetic materials that causes them to change their shape or dimensions during the process of magnetization. The variation of material's magnetization due to the applied magnetic field changes the magnetostrictive strain $\stackrel{\leftrightarrow}{\in}$ until reaching its saturation value, $\underline{\lambda}$, described by

$$
\vec{H}=\stackrel{\leftarrow 3}{X}: \overleftrightarrow{\epsilon}
$$

where $\stackrel{\leftrightarrow 3}{X}$ is the third-rank inverse magnetostrictive strain triadic, which is also centrosymmetric reducing its 27 components to 18 independent values for general isotropic ferromagnetic materials. Much like piezoelectric materials, Magnetostriction is also a reversible process in that applying tension or compression to the ferroelectric material created an internal magnetic field.

With these two examples we introduce rank-three tensors to the undergraduate explaining that the physics of piezoelectric and magnetostrictive effects is left to separate courses in coupled systems electromechanics and our purpose here is to get them ready to deal with even higher-ranked tensors without becoming overwhelmed with the tedious character of the expansions. We do however point out that the detailed expansion of the dyadic as detailed in Eq.(1-4) becomes three such expansions making up 27 components of the form

$$
\left(\begin{array}{c}
\stackrel{\leftrightarrow 3 \rightarrow}{\Psi} \\
\stackrel{\leftrightarrow 3 \rightarrow}{\mathrm{X}}
\end{array}\right)=\sum_{i=1}^{3} \sum_{j=1}^{3} \sum_{k=1}^{3} \hat{u}_{i} \hat{u}_{j} \hat{u}_{k}\left(\begin{array}{c}
\psi_{i j k} \\
\chi_{i j k}
\end{array}\right)=\sum_{i=1}^{3} \sum_{j=1}^{3} \sum_{k=1}^{3} u_{i j k}\left(\begin{array}{c}
\psi_{i j k} \\
\chi_{i j k}
\end{array}\right)
$$

where the combination of the unit vectors $\hat{u}_{i} \hat{u}_{j} \hat{u}_{k}$ without dot or cross product operations implies tensor product operations denoted by $\otimes$ as $\hat{u}_{i} \otimes \hat{u}_{j} \otimes \hat{u}_{k}$ simplified by the triple-hatted unitary triadic $u_{i j k}$, which can be referred to as a $\operatorname{triad}^{8}$. 


\section{Example 3. Photoelasticity phenomena-Introducing the four-rank tensor}

Photoelasticity phenomena is related to birefringence of the thin polymeric film put on the surface of the body subjected to some stresses. Birefringence of the film is related to the anisotropy of dielectric permittivity as a result of orientation of macromolecules differently stretched in different direction. Formally, the film dielectric permittivity dyadic $\overleftrightarrow{\varepsilon}$ can be linearly linked to the stress $\vec{\sigma}$ in the body with the four-rank photoelectric stress tensor

$$
\vec{\varepsilon}=\stackrel{\leftarrow \leftrightarrow}{\Xi}: \vec{\sigma}
$$

where the four-rank tensor is expanded as

$$
\stackrel{\leftarrow \leftrightarrow}{\Xi}=\sum_{i=1}^{3} \sum_{j=1}^{3} \sum_{k=1}^{3} \sum_{\ell=1}^{3} \hat{u}_{i} \hat{u}_{j} \hat{u}_{k} \hat{u}_{\ell} \xi_{i j k \ell}=\sum_{i=1}^{3} \sum_{j=1}^{3} \sum_{k=1}^{3} \sum_{\ell=1}^{3} u_{i j k \ell} \xi_{i j k \ell}
$$

Although this tensor has in general 81 components it's symmetry reduces that number to 36 because of the symmetry of both the stress dyadic and the permittivity dyadics. Still the notation is tedious and can be simplified as

$$
\Xi_{\mathrm{ijk} \ell}
$$

where all of the summations, unit vectors and the unitary tensor with the four hats are suppressed for simplicity of notation, but understood as a necessary part of performing actual analyses of photoelastic phenomena where in this case a rank-four tensor was necessary.

\section{Example 4. Coupled Systems Mechanics-Thermoelastic- Piezoelectric-Magnetostrictive Effects}

The generalized linear law for coupled effects can be expressed as

$$
\overleftrightarrow{\epsilon}=\overleftrightarrow{\alpha} \Delta T+\stackrel{\leftrightarrow 3}{\Pi} \cdot \vec{E}+\stackrel{\leftrightarrow 3}{\Theta} \cdot \vec{H}+\stackrel{\leftarrow 4}{C}: \overleftrightarrow{\sigma}
$$

where the strain $\overleftrightarrow{\in}$, stress $\vec{\sigma}$, electric and magnetic field intensities $\vec{E}$ and $\vec{H}$ are related by the thermal-strain dyadic $\vec{\alpha}$, the piezoelectric strain triadic $\stackrel{\leftarrow 3}{\Pi}$, the magnetostrictive strain triadic $\stackrel{\leftrightarrow 3}{\Theta}$, and the compliance tensor $\stackrel{\leftarrow}{C}$.

In general Eq. (4-1) represents a system of nine linear equations and nine independent vaariables. In order to determine the inverse of (4-1), i.e. to obtain $\vec{\sigma}$ as a function of $\Delta T, \vec{E}, \vec{H}$ and $\vec{\in}$, we multiply each term of (41) by the inverse of the compliance tensor, and solve for $\vec{\sigma}$, which becomes

$$
\vec{\sigma}=-(\stackrel{\leftarrow 4 \rightarrow}{C})^{-1}: \alpha \Delta T-(\stackrel{\leftarrow 4 \rightarrow}{C})^{-1}:(\stackrel{\leftrightarrow 3 \rightarrow}{\Pi \cdot \vec{E}})_{-}(\stackrel{\leftarrow 4 \rightarrow}{C})^{-1}:(\stackrel{\leftarrow 3 \rightarrow}{\Theta} \cdot \vec{H})_{+}(\stackrel{\leftarrow 4 \rightarrow}{C})^{-1}: \vec{\epsilon}
$$


Therefore the inverse of (4-1) is expressed as

$$
\overleftrightarrow{\sigma}=\overleftrightarrow{\beta} \Delta T+\stackrel{\leftarrow}{\Lambda} \cdot \vec{E}+\stackrel{\leftarrow 3}{\Gamma} \cdot \vec{H}+\stackrel{\leftarrow 4}{S}: \overleftrightarrow{\epsilon}
$$

Where $\overleftrightarrow{\beta}$ is the thermoelastic dyadic given by

$$
\overleftrightarrow{\beta}=-(\stackrel{\leftarrow \rightarrow}{C})^{-1}: \overleftrightarrow{\alpha}
$$

$\stackrel{\leftarrow 3}{\Lambda}$ is the piezoelectric stress triadic given by

$$
\stackrel{\leftrightarrow 3}{\Lambda}=-(\stackrel{\leftarrow 4 \rightarrow}{C})^{-1}: \stackrel{\leftrightarrow 3}{\Pi}
$$

$\stackrel{\leftrightarrow 3}{\Gamma}$ is the magnetostrictive stress triadic given by

$$
\stackrel{\leftarrow 3 \rightarrow}{\Gamma}=-(\stackrel{\leftarrow \rightarrow}{C})^{-1}: \stackrel{\leftarrow 3}{\Theta}
$$

and $\stackrel{\leftarrow 4}{S}$ is the stiffness tensor, which is the inverse of the compliance tensor

$$
\stackrel{4}{S}=(\stackrel{\leftarrow 4 \rightarrow}{C})^{-1}
$$

For the undergraduate student we explain why the first coefficient is a dyadic, the second and third coefficients are triadics and the fourth coefficient is a tensor of rank 4 (also known as a quadadic) by the explanation given in Maxum (Reference 1, Section 3.7).

In the cases where the electric and magnetic fields vanish, we have from (4-1)

$$
\overleftrightarrow{\epsilon}=\overleftrightarrow{\alpha} \Delta T+\stackrel{4}{C}: \overleftrightarrow{\sigma}
$$

and for zero stress the strain is $\vec{\epsilon}=\vec{\alpha} \Delta T$, which means that each of its nine components are given by the thermalstrain dyadic $\vec{\alpha}$ components as a function of thermal change. Similarly, where the fields vanish, the stress is

$$
\overleftrightarrow{\sigma}=\overleftrightarrow{\beta} \Delta T+\stackrel{\leftarrow 4}{S}: \overleftrightarrow{\epsilon}
$$

and for zero strain the stress is $\vec{\sigma}=\overleftrightarrow{\beta} \Delta T$, which means that each of its components are given by the thermalstress dyadic $\overleftrightarrow{\beta}$ as a function of thermal change. 


\section{Example 5. Constitutive laws of nonlinear optics}

The nonlinear constitutive relation between the electric flux density field $\vec{D}$ (also commonly referred to as the displacement vector field) and the electric field intensity field $\vec{E}$ is an extension of Eq. (1-1) into higher ranked permittivity tensors $\vec{\varepsilon}, \stackrel{\leftarrow 3 \rightarrow}{\varepsilon}, \stackrel{\leftarrow 4}{\varepsilon}, \cdots$ and the higher tensor products of the $\vec{E}$ field are $\vec{E}^{2} \equiv[\vec{E} \otimes \vec{E}]$, and $\vec{E}^{3} \equiv[\vec{E} \otimes \vec{E} \otimes \vec{E}]$, etc., as

$$
\begin{aligned}
\vec{D} & =\vec{\varepsilon} \cdot \vec{E}+\stackrel{\leftarrow 3 \rightarrow}{\varepsilon}: \vec{E}^{2}+\stackrel{\leftarrow 4}{\varepsilon}: \vec{E}^{3}+\cdots \\
& =\vec{\varepsilon} \cdot \vec{E}+\stackrel{\leftarrow 3}{\varepsilon}:[\vec{E} \otimes \vec{E}]+{ }^{\leftarrow 4} \varepsilon:[\vec{E} \otimes \vec{E} \otimes \vec{E}]+\cdots
\end{aligned}
$$

At this point we introduce to the undergraduate the concept of the tensor product $\otimes$, pointing out that there are three types of vector-vector products, namely the dot product "." , the cross product " $\times$ " , of which they are already familiar, and the tensor product as just introduced above.

Another approach to nonlinear optics is to expand the polarization vector $\vec{P}$ rather than $\vec{D}$.

In linear optics of crystals the susceptibility dyadic $\vec{\chi}$ is used for linking the polarization density vector $\vec{P}$ with the electrical field intensity $\vec{E}$ as $\vec{P}=\varepsilon_{0} \vec{\chi} \cdot \vec{E}$. In nonlinear optics this is expressed in terms of susceptibility tensors of increasing ranks where the powers of $\vec{E}$ are defined by $\vec{E}^{2} \equiv[\vec{E} \otimes \vec{E}]$ and $\vec{E}^{3} \equiv[\vec{E} \otimes \vec{E} \otimes \vec{E}]$, which are rank two and three tensors, respectively.

As is shown in Field Mathematics ${ }^{9}$

- The tensor (direct) product of two tensors yields another tensor of rank equaling the sum of the ranks of the two tensors,

- The cross product of two tensors yields a tensor of rank one less than the sum of the ranks of the two tensors.

- The dot product of two tensors yields a tensor of rank two less than the sum of the ranks of the two tensors,

- The multiple dot product of two tensors yields a tensor of rank equalling the sum of the two tensors less two for each multiple dot product.

Notice that in the last term of $(5-1), \stackrel{\leftarrow 4 \rightarrow}{\varepsilon}$ is rank 4 and $[\vec{E} \otimes \vec{E} \otimes \vec{E}]$ is rank 3, thus the sum of the ranks of the two tensors is seven $(3+4=7)$. Then applying the fourth bullet above to the triple product we subtract $2 \times 3=6$ and obtain one for the rank of $\stackrel{\leftarrow 4 \rightarrow}{\varepsilon}:[\vec{E} \otimes \vec{E} \otimes \vec{E}]$ which is a vector. Likewise in the second term we get $3+2-(2 \times 2)=1$, and the first term we get $2+1-2=1$, resulting in a vector for each term , because every term of every equation must have the same rank ${ }^{10}$. 
In isotropic material such as glass the polarization density and the electrical field intensity are collinear. In this case the constitutive relation for the nonlinear optical phenomena isotropic body is ${ }^{11}$

$$
\vec{P}=\varepsilon_{0}\left[\overleftrightarrow{\chi} \cdot \vec{E}+\stackrel{\leftarrow 3}{\chi}: \vec{E}^{2}+\stackrel{\leftarrow 4}{\chi}: \vec{E}^{3}+\cdots\right]
$$

where susceptibilities of increasing rank are introduced. The generalization to the nonlinear case can be made formally in three ways:

(a) The susceptibility components in terms of the electric field intensity:

$$
\vec{P}=\varepsilon_{0}\left[\overleftrightarrow{\chi}\left(1+\alpha_{1}|E|+\alpha_{2}|E|^{2}+\alpha_{3}|E|^{3}+\cdots\right)\right] \cdot \vec{E}
$$

where $E^{2}$ is the scalar $\vec{E} \cdot \vec{E}$ and $\overleftrightarrow{\chi}()$ is a functional relationship.

(b) The susceptibility components in terms of the electric field intensity vector and susceptibility dyadic:

$$
\begin{aligned}
\vec{P} & =\varepsilon_{0} \overleftrightarrow{\chi}(W) \cdot \vec{E} \\
& =\varepsilon_{0} \overleftrightarrow{\chi}\left(1+\beta_{1} \sqrt{W}+\beta_{2} W+\beta_{3} W \sqrt{W}+\cdots\right) \cdot \vec{E}
\end{aligned}
$$

where $W=[\vec{\chi} \cdot \vec{E}] \cdot \vec{E}$ is the invariant of the susceptibility dyadic and the electric field intensity vector.

(c) The susceptibility tensors of increasing ranks:

$$
\vec{P}(t)=\varepsilon_{0}\left\{\begin{array}{l}
\overleftrightarrow{\chi} \cdot \vec{E}(t)+\stackrel{\leftarrow}{\chi}:[\vec{E}(t) \otimes \vec{E}(t)] \\
\leftarrow 4 \rightarrow:[\vec{E}(t) \otimes \vec{E}(t) \otimes \vec{E}(t)]+\cdots
\end{array}\right\}
$$

The difference between these approaches is in the effects on the polynomial coefficients connecting each component of the vector $\vec{P}$ to each component of the vector $\vec{E}$ of the change in angle with respect to the axes of the crystal. In the relaxing media, the vector $\vec{P}$ depends not only on the vector $\vec{E}$ at time $t$, but also from the previous time history of the vector $\left.\vec{E}(\tau)\right|_{-\infty} ^{t}$. This would lead us to the concept of tensor-functionals, which is not a topic of current presentation for undergraduates. This and other such topics are being left to future work. 


\section{Summary}

These examples show the importance of understanding the tensor nature of many objects and phenomena in modern physics and engineering, thus demonstrating why tensors need to be studied at undergraduate levels by first exposing dyadics (tensors of rank two) as shown in Example 1; then triadics (tensors of rank three) in Example 2; followed by Example 3 which introduces a tensor of rank four. In Example 4 we show how the undergraduate is introduced to coupled systems that use tensors of ranks 2, 3, and 4. And finally we show infinites series of tensors of ever increasing ranks in Example 5. This approach has been successfully done for many years at some advanced universities. However, the authors' practice has shown that it can be successfully presented even in rural universities, such as Lamar University (Beaumont, TX) and Pittsburg State University (Pittsburg, KS) with students that come without the sophistication typically found at the ivy league schools.

\footnotetext{
${ }^{1}$ Maxum, Bernard, Field Mathematics for Electromagnetics, Photonics and Materials Science, SPIE Press, Fourth printing, 2007 \{ISBN 081945523-7\}, SPIE - The International Society for Optical Engineering, P.O. Box 10, Bellingham, WA, USA.

2 Ibid., Section 2.3.

${ }^{3}$ Ibid., Section 3.3 and in particular pages 3-6 and 3-7 with Eq. (3.4-4).

${ }^{4}$ Piezoelectric effect from Wikipedia (http://en.wikipedia.org/wiki/Piezoelectricity\#Mechanism).

${ }^{5}$ Inversion symmetry, from Wikipedia

(https://www.google.com/search?q=inversion+symmetry\&rlz=1C1GGGE en US456\&oq =\&aqs=chrome.3.69i58j0i66|3j5i66I2.47144681j0j0\&sourceid=chrome\&es sm=93\&ie=UTF-8.)

${ }^{6}$ Maxum, op. cit, last paragraph of pg.3-13 and Table 3-1, pg. 3-22.

${ }^{7}$ Magnetostriction from Wikipedia (http://en.wikipedia.org/wiki/Magnetostriction).

${ }^{8}$ Maxum, op. cit., last paragraph of Pg. 3-13.

${ }^{9}$ Maxum, op. cit. , Sections 3.4, 3.6, 3.7, 3.8 and Table 3-1.

${ }^{10}$ Maxum, op. cit.., Section 3.7.

11 Agrawal, Govind P., Nonlinear Fiber Optics, Elsevier, Inc. 2007 (ISBN 13: 978-0-12-369516-1), Section 2.3.
} 